\title{
REPORT OF THE INTERNATIONAL WORKSHOP ON VIRTUAL REALITY AND APPLICATIONS
}

\author{
Jiaoying Shi \\ Co-chair of the workshop \\ Zhejiang University, P.R. China \\ Zhigeng Pan \\ Content editor for IJVR \\ Hong Kong Polytechnic University
}

\section{Purpose}

The International Workshop on Virtual Reality and Application (VR\&A) was held in Zhejiang University, China in June, 1999. The purposes of this workshop were as follows:

(1) To provide a forum for experts, researchers and developers from all over the world to present their latest research results. This international workshop followed on from the success of the first international workshop on VR and Visualization in Scientific Computing, held at the same location in 1995.

(2) 1999 was the 30th anniversary of SIGGRAPH, the 25th anniversary of Eurographics, and the 10th anniversary of the State Key Lab of Computer Aided Design and Computer Graphics (CAD\&CG), Zhejiang University. The event served to celebrate these events.

(3) The State Key Lab of CAD\&CG, Zhejiang University had established a four-screen CAVE, which is the first in China. A ceremony to open the CAVE was held during the international workshop.

\section{Location and Dates}

The international workshop was held over three days, June 20-22, 2000. The opening speeches were given by Prof. Yunhe Pan (President of Zhejiang University), Dr. Martin Göebel (Director of VMSD, IMK-GMD, Germany, co-chairman of the workshop), Prof. Jiaoying Shi (Director of Academic Committee of State Key Lab. of CAD\&CG, Co-chairman of the workshop), and Prof. Jinyeu Tsou (The Chinese University of Hong Kong, CUHK for short) (See Fig. 1-4).

The workshop closed with an academic discussion on the future development of VR chaired by Prof. Mark Green and Prof. Enhua Wu. After the workshop, the organizing committee arranged a sight-seeing trip to Zhou Zhuang located in Kunshan city, China. 


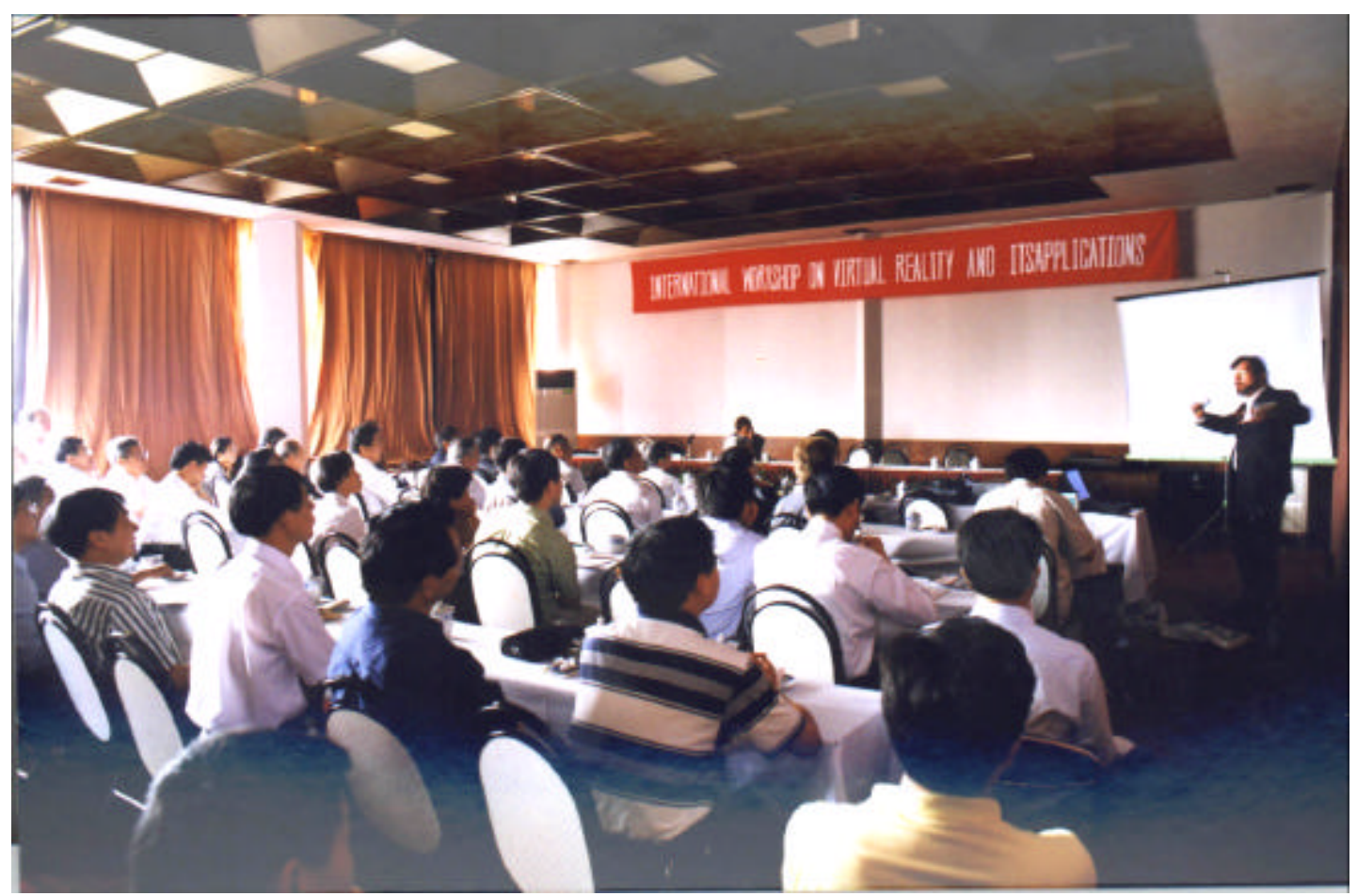

Fig. 1 Attendees at a presentation

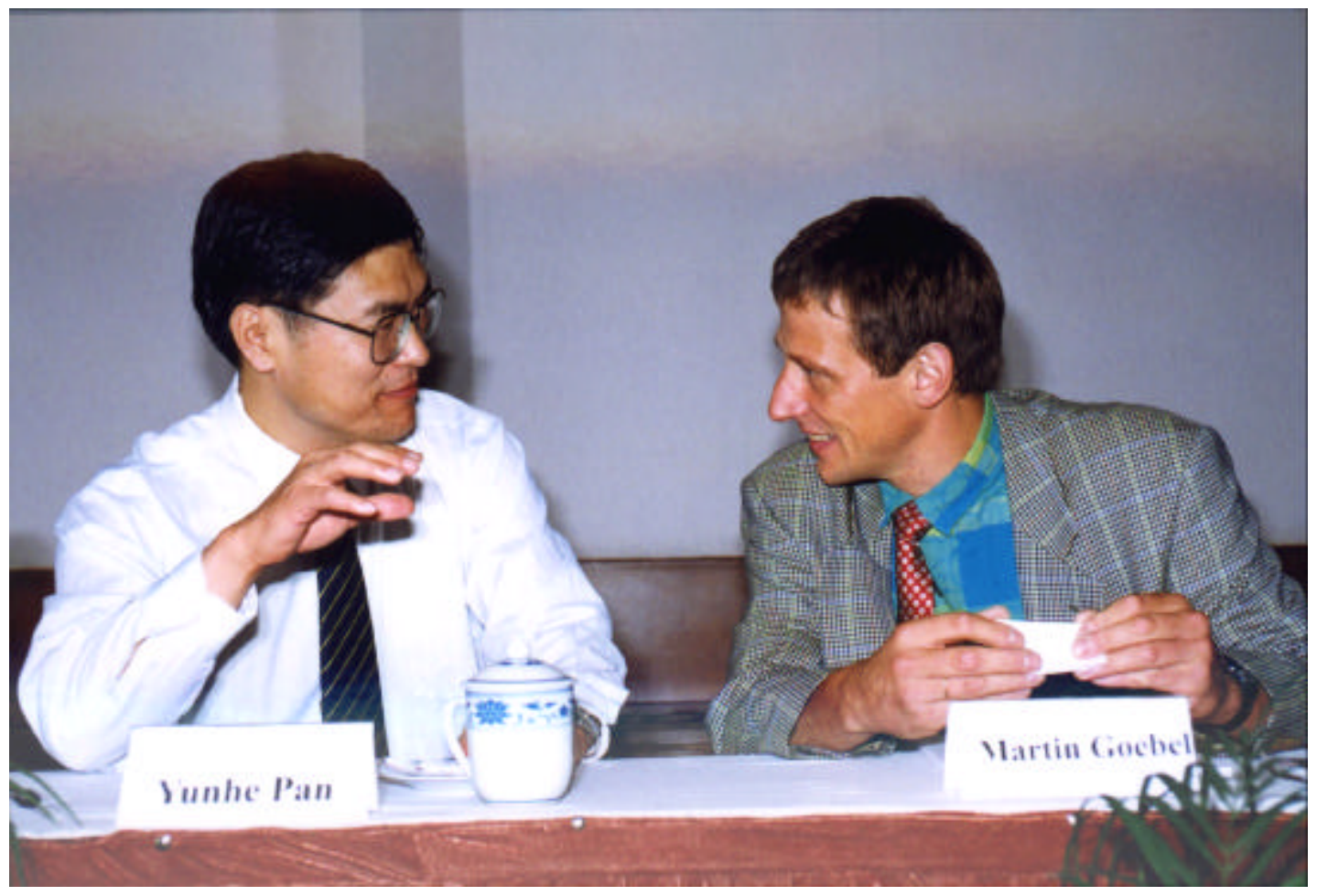

Fig. 2 Prof. Yunhe Pan (President of Zhejiang University) and Dr. Martin Goebel (Co-chairman of the workshop) 


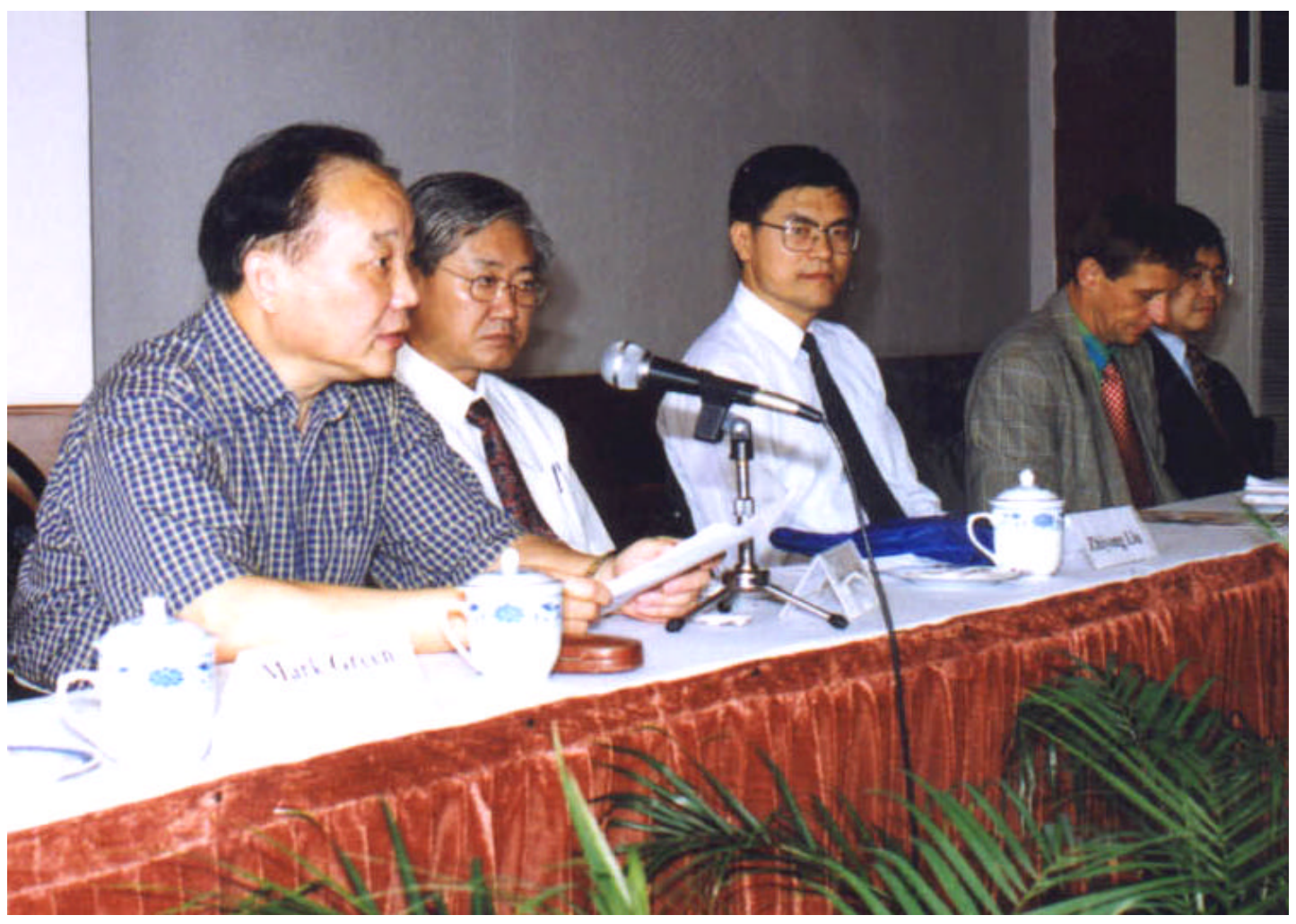

Fig. 3 Prof. Jiaoying Shi (Co-chairman of the workshop)

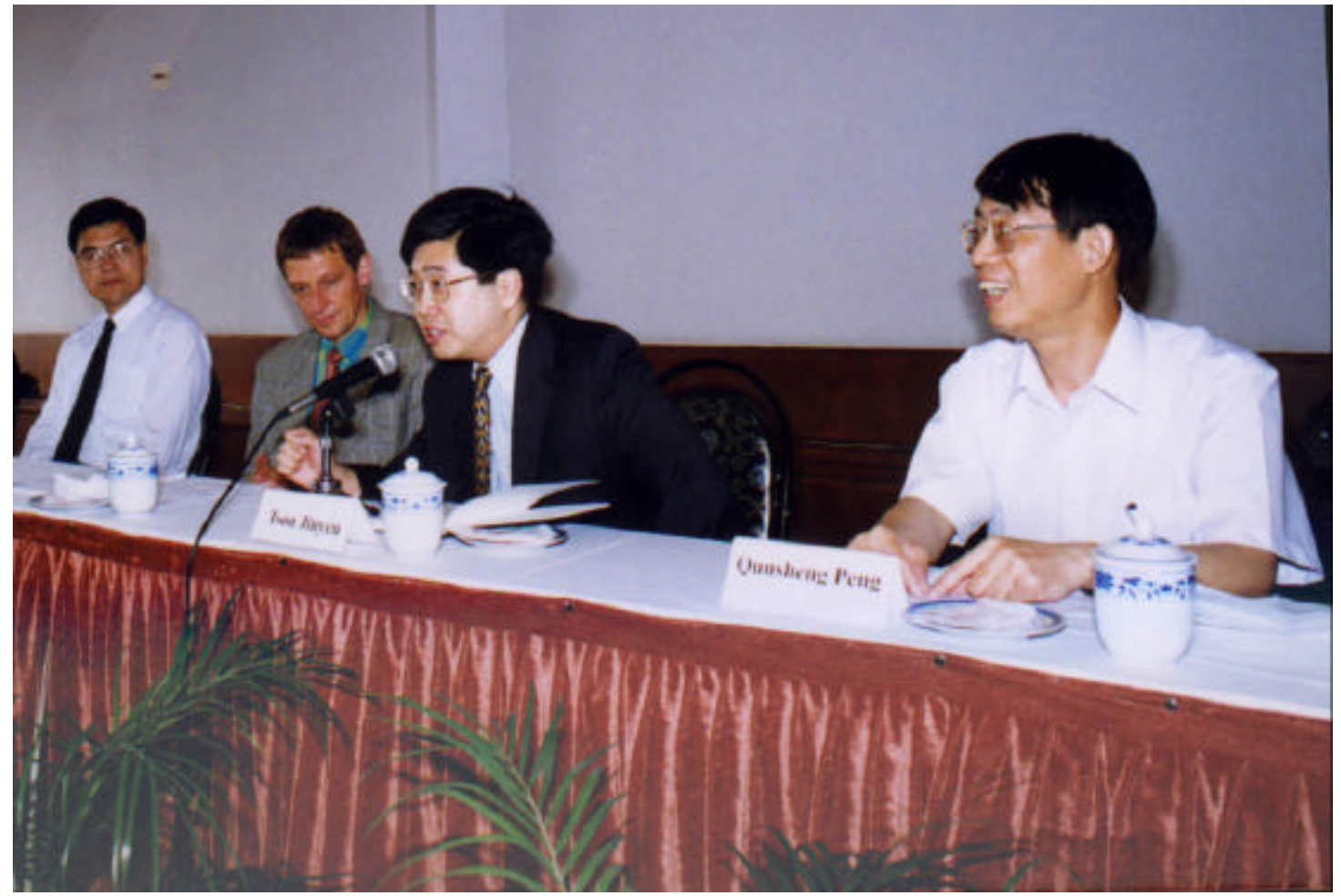

Fig. 4 Prof. Jinyeu Tsou (CUHK) and Prof. Qunsheng Peng (Chairman of the organizing committee) 


\section{Workshop Attendees and General Themes}

The workshop attracted more than 60 attendees, most from the mainland of China. Others came from the U.S.A, Canada, Germany, Japan, Singapore, Hong Kong, and Macao. The Chinese participants are mainly from universities (Tsinghua University, Nanjing University, Zhejiang University, Changsha Polytechnic University, East China Polytechnic University, North Jiaotong University, Harbin University of Technology, etc), from institutes of the Chinese Academy of Sciences (Institute of Computing Technology, Institute of Software, Institute of Automation), and from industrial companies (Real Time Graphics Inc., Beijing, Bell Company in Shanghai). A number of Ph.D. and M.S students attended as well.

The general themes of the workshop include:

- Theories and algorithms of virtual reality

- Software issues of a CAVE system

- Distributed virtual reality

- Collaborative virtual environment

- Virtual acoustical environment

- Applications of VR in scientific visualization

- Applications of VR in architecture

- Applications of VR in medical visualization

- Applications of VR in mechanical engineering

\section{Plenary Talks}

Some VR experts were invited to deliver plenary presentations during on the workshop. The topics for the talks are listed below, detail information of the talks is included in the accompanying CD-ROM of this special issue:

The Challenge of 3D Interaction, or Why Is 3D Interaction So Hard? (Prof. Mark Green, University of Alberta in Canada)

Realtime Visual Scene Management and Simulation System for Distributed Virtual Environment on PCs and Its Applications (Prof. Jian Wang, Zhejiang University)

Virtual Environments - from Visualization to Collaboration (Dr. Martin Göebel, GMD - German National Research Center for Information Technology)

Virtual Acoustical Environment (Prof. Jiaoying Shi, Zhejiang University)

Virtual Simulation towards Empowering Design Creativity and Building Construction (Prof. Jinyeu Tsou, The Chinese University of Hong Kong)

Rendering of Landscapes for Environmental Assessment (Prof. Eihachiro Nakamae, Hiroshima University, Japan)

Multi-Resolution Modeling and Its Application in VR (Prof. Zhigeng Pan, Hong Kong Polytechnic University, Zhejiang University)

Virtual Bronchoscopy (Dr. Pheng-ann Heng, The Chinese University of Hong Kong)

Industrial Applications of Immersive Virtual Reality (Dr. Hong-Yee Wong, Institute of High Performance Computing, Singapore)

Virtual Reconstruction of Church St. Paulo from its Ruins (Prof. Enhua Wu, The University of Macao) Ophthalmic Visualization and Virtual Surgery (Prof. Brain Barsky, The University of California, Berkeley)

Virtual Simulation of Radiotherapy Treatment Planning (Mr. Wenli Cai, Fraunhofer Institute for Computer Graphics, Germany) 


\section{Opening Ceremony of CAVE}

An Opening Ceremony of CAVE (Cave Automatic Virtual Environment) was held in front of Teaching Building no.1 in the morning of June 21. Speeches were delivered by Prof. Yunhe Pan (President of Zhejiang University), Ms. Chengchen Yuan (Deputy director, Dept. of Science and Technology, the Ministry of Education), Prof. Zhiyong Liu (Deputy Director of Information Division, National Natural Science Foundation of China), Prof. Xiaolang Yan (Director of Committee of Science and Technology, Zhejiang Province), Dr. Martin Göebel (Director, VMSD, IMK-GMD, Germany), and Prof. Mark Green (University of Alberta in Canada). After the ceremony, workshop attendees visited the CAVE environment, and saw very interesting demos brought from GMD in Germany and University of Albert in Canada. The software system AVOCARDO used for the first CAVE in China was given by the GMD of Germany under the support by the Int'l Cooperative Beauro of the Ministry of Science, Technology and Education of Germany. Special thanks go to Dr. Koepke, the director of the Beauro, and Mrs. Hongsernant.

\section{Introduction to the Workshop Chairs}

Dr. Martin Göebel is the Head of the Virtual Environments Research Division in the Institute for Media Communication of the German National Research Center for Information Technology (GMD). $\mathrm{He}$ is responsible for R\&D projects in high performance interactive Scientific Visualization, Virtual Studios, Simulation and Virtual Environments. He established and coordinated the application-oriented Fraunhofer Demonstration Centers for Virtual Reality in Darmstadt and Stuttgart from 1993 to 1995. In 1996, he joined GMD where he developed the first European CAVE-like-set-up in Bonn. Dr. Martin Göebel received the PhD (Dr.-Ing.) in 1990 from Darmstadt University. He is the author and editor of several books on Graphics Standards, Visualization and Virtual Reality. He has chaired four Eurographics Workshops on Virtual Environments from 1993 to 1998. He has been the program cochair of the EUROGRAPHICS 95 and 98 conference, the conference co-chair of the Virtual Enviromnments'98 conference and has been program committee member of many Conferences in VR and visualization for years. Martin Göebel is a member of the IEEE Computer Society, the EUROGRAPHICS Association and the German Computer Society (GI). He is member of the editorial advisory board of the international journal Computers \& Graphics.

Jiaoying Shi is a professor of the Department of Computer Science and Engineering at Zhejiang University which is located in Hangzhou, Zhejiang Province of China. He is now the Director of Academic Committee of State Key Lab of Computer Aided Design and Computer Graphics (in short as State Key Lab of CAD\&CG). Prof. Shi is the Deputy Chairman of China Image and Graphics Association, the Deputy Chairman of China CAD and Graphics Society under China Computer Federation. He is a member of SIGGRAPH Education Committee, and on the Editorial Board of international journal Computers \& Graphics. He served as the Vice Chairman of the Department of Computer Science and Engineering(1985-1988), the Director of University Division of Scientific Research Affairs (1988-1991), and the Director of State Key Lab of CAD\&CG for 10 years ( 1989-1998 ). In late 1980s' he was actively involved in the research work on computer graphics area. Since 1990 his work is concentrated on visualization in scientific computing and virtual environment. He has published more than 100 papers and two books. 


\section{Closing Comments}

The workshop is a success, and it will certainly enlarge and extend the research and development of VR in China.

We would like to express our sincere thanks to the Committee of the National Natural Science Foundation of China for its fund support, IJVR journal and IPI Press for publishing talks and related materials presented on this Workshop. We would also like to express our sincere thanks to Dr. Martin Göebel, [] the co-chair of both international workshops, for his firm support to us in the past few years, and especially for sending experts to our lab to install AVOCARDO software which is a VR development platform developed by his people at GMD, and many wonderful demos developed by his group too.

Finally, we hope we can make promising achievements in the field of VR and its application, and we may organize the third workshop in this direction in three or four years. 